\title{
Erratum to: Mathematical Justification of the Obstacle Problem in the Case of a Shallow Shell
}

\author{
Alain Léger · Bernadette Miara
}

Received: 1 November 2009 / Published online: 5 December 2009

(C) Springer Science+Business Media B.V. 2009

Erratum to: J Elasticity (2008) 90: 241-257

DOI 10.1007/s10659-007-9141-1

This correction concerns the statement of Theorem 5.2, page 250, and Remark 4, page 255.

(1) The correct statement of point 3 of Theorem 5.2 is:

The function $\zeta=\left(\zeta_{\alpha}, \zeta_{3}\right)$ solves the following problem:

$$
\begin{aligned}
& \text { Find }\left(\zeta_{\alpha}, \zeta_{3}\right) \in \boldsymbol{V}_{H}(\omega) \times K_{3}(\omega) \\
& -\int_{\omega} m_{\alpha \beta}\left(\zeta_{3}\right) \partial_{\alpha \beta}\left(\eta_{3}-\zeta_{3}\right) d \omega+\int_{\omega} n_{\alpha \beta}^{\theta}(\zeta) \partial_{\alpha} \theta \partial_{\beta}\left(\eta_{3}-\zeta_{3}\right) d \omega \\
& \quad \geq \int_{\omega} p_{3}\left(\eta_{3}-\zeta_{3}\right) d \omega+\int_{\omega} s_{\alpha} \partial_{\alpha}\left(\eta_{3}-\zeta_{3}\right) d \omega, \quad \forall \eta_{3} \in K_{3}(\omega), \\
& \int_{\omega} n_{\alpha \beta}^{\theta}(\zeta) \partial_{\beta} \eta_{\alpha} d \omega=\int_{\omega} p_{\alpha} \eta_{\alpha} d \omega, \quad \forall \eta_{H}=\left(\eta_{\alpha}\right) \in \boldsymbol{V}_{H}(\omega) .
\end{aligned}
$$

This correction does not affect the proof of the theorem.

(2) Remark 4 must be changed into:

The online version of the original article can be found under doi:10.1007/s10659-007-9141-1.

A. Léger $(\varangle)$

Laboratoire de Mécanique et d'Acoustique, CNRS, 31, chemin Joseph Aiguier, 13402 Marseille Cedex 20, France

e-mail: leger@1ma.cnrs-mrs.fr

B. Miara

Laboratoire de Modélisation et Simulation Numérique, École Supérieure d’Ingénieurs en Électrotechnique et Électronique, Cité Descartes, 2 Boulevard Blaise Pascal, 93160 Noisy-le-Grand Cedex, France

e-mail: b.miara@esiee.fr 
Remark 4 Problem (19) is a couple problem. It is interesting to observe that the coupling arises from the resultant $n_{\alpha \beta}^{\theta}(\zeta)$ which depends both on the inplane components $\zeta_{\alpha}$ and on the transverse component $\zeta_{3}$ of the displacement of the middle surface of the shallow shell. On the one hand the variational equality is the classical equilibrium equation of the resultant, which is the same as the one of the bilateral case. On the other hand, the variational inequality involves both the bending momentum $m_{\alpha \beta}\left(\zeta_{3}\right)$ which depends only on the transverse component $\zeta_{3}$ and the resultant which depends on the three components of $\zeta$. 